\title{
BMJ Open Anticholinergic burden and fractures: a protocol for a methodological systematic review and meta-analysis
}

\section{Jonas Reinold, ${ }^{\oplus 1}$ Wiebke Schäfer, ${ }^{1}$ Lara Christianson, ${ }^{1}$ Francesco Barone-Adesi, ${ }^{2}$} Oliver Riedel, ${ }^{1}$ Federica Edith Pisa ${ }^{1}$
To cite: Reinold J,

Schäfer W, Christianson L, et al. Anticholinergic burden and fractures: a protocol for a methodological systematic review and meta-analysis. BMJ Open 2019;9:e030205. doi:10.1136/ bmjopen-2019-030205

- Prepublication history and additional material for this paper are available online. To view please visit the journal (http:// dx.doi.org/10.1136/bmjopen2019-030205).

Received 04 March 2019 Revised 19 July 2019

Accepted 02 August 2019

\section{Check for updates}

(c) Author(s) (or their employer(s)) 2019. Re-use permitted under CC BY-NC. No commercial re-use. See rights and permissions. Published by BMJ.

${ }^{1}$ Leibniz Institute for Prevention Research and Epidemiology BIPS, Bremen, Germany

${ }^{2}$ Department of Pharmaceutical Sciences, University of Eastern Piedmont, Novara, Italy

Correspondence to Federica Edith Pisa; pisa@leibniz-bips.de

\section{ABSTRACT}

Introduction Medications with anticholinergic activity are used in the treatment of many diseases common in old age, including depression, psychosis, Parkinson's disease, allergies, pain and urinary incontinence. A high anticholinergic burden (ACB) is considered a major risk factor for fractures in older adults but recent studies reported inconsistent results. These inconsistencies may partly be due to differences in methodological aspects. However, no systematic review so far has addressed this association and considered study methods. Thus, we aim to conduct a systematic review and meta-analysis of observational studies addressing the association of ACB with fractures and to provide a methodological appraisal of the included studies.

Methods and analysis We will search MEDLINE, EMBASE, the Science Citation Index, CENTRAL and grey literature using a strategy that combines the terms anticholinergic and fractures. We will hand search reference lists of articles. Two reviewers will independently screen all identified abstracts for eligibility and evaluate the risk of bias of the included studies using the Newcastle-Ottawa Quality Assessment Scale and RTI item bank. Discrepancies will be resolved by consensus or consultation with a third researcher. We will conduct a meta-analysis, either for the overall population or for specific and more homogeneous subgroups, if the number of studies retrieved and their heterogeneity allows it. Ethics and dissemination No ethics approval will be sought, as no original data will be collected for this review. Findings will be disseminated through peer-reviewed publication and conference presentations.

PROSPERO registration number CRD42018116737.

\section{BACKGROUND}

Several studies have found that older adults who concurrently use multiple medications with anticholinergic activity (MACs) are at increased risk of adverse health outcomes. These outcomes include fractures, which greatly affect older patients' health and quality of life. ${ }^{1-4}$ However, conclusive evidence on this association and on its magnitude is still lacking.

MACs encompass drugs with a wide range of indications, such as agents for overactive bladder (oxybutynin), H2 antagonists

\section{Strengths and limitations of this study}

Development of search algorithm guided by experienced librarian.

- Search for eligible articles will be conducted in four large databases for scientific publications, various sources for grey literature, references of included studies as well as studies citing included studies.

- No language restrictions will be applied.

- Two commonly used risk assessment tools for observational studies will be applied, thus, increasing the depth of risk assessment but also complexity of interpretation.

(ranitidine, cimetidine), bronchodilators (theophylline), antiparkinsonians (orphenadrine), antipsychotics (clozapine, quetiapine) and antidepressants (amitriptyline, paroxetine). Their anticholinergic activity is due to the competitive inhibition of muscarinic receptors in the central nervous system and peripheral tissues. There are MACs with high anticholinergic activity (eg, amitriptyline, oxybutynin, ipratropium), while others display only weak activity (eg, digitoxin, ranitidine, olanzapine) ${ }^{5-7}$ Adverse reactions often result from the cumulative burden of MACs taken by a patient. ${ }^{8}$ This cumulative exposure is defined as anticholinergic burden (ACB). Several tools have been developed to measure the ACB based on patients' medication lists. ${ }^{14-12}$ With the exception of the anticholinergic component of the Drug Burden Index (DBI-Ach), ${ }^{13}$ all tools assign each MAC a score based on its anticholinergic activity. The sum of scores of all MACs concurrently taken by a patient determines his/her ACB score. The DBI-Ach ${ }^{13}$ and the Muscarinic Acetylcholinergic Receptor ANTagonist Exposure scale (MARANTE) ${ }^{11}$ are however the only tools that account for the dose, an important dimension of the exposure to MACs. 
In older adults, a high ACB has been associated with an increased risk of cognitive and functional impairment, ${ }^{14-25}$ visual acuity ${ }^{26}$ as well as severe adverse outcomes, ${ }^{1} 24619$ 26-38 including fractures. ${ }^{39-41}$ The ACB has been consistently associated with an increased risk of falls that are by large the main cause of fractures in older adults, ${ }^{42} 43$ and several MACs have been considered fall-risk increasing medications. ${ }^{44-46}$ Moreover, a recent study showed that high ACB is associated with low bone mineral density in middle-aged and older women. ${ }^{47}$ However, very limited evidence on this relation is available so far.

In this systematic review, we have chosen to focus on fractures as an outcome for the following reasons: (i) fractures are clinically relevant outcomes greatly affecting older patients' health and quality of life, leading to reduced autonomy, ${ }^{48-50}$ increased morbidity, short-term mortality ${ }^{5152}$ and healthcare costs ${ }^{53-55}$; (ii) in claims and other administrative healthcare databases, non-injurious falls are generally not well captured; (iii) there are already systematic reviews that have addressed the risk of falls associated with the use of anticholinergic medication, ${ }^{1305657}$ but none have yet focused on the association of high ACB and fractures. Given that an effect of the ACB on bone density could have important clinical implications, we will also assess whether studies that addressed fractures as an outcome also evaluated osteoporosis and/ or bone density.

Sound evidence on modifiable risk factors is thus needed to identify targets for prevention. Despite the relevance of fractures for older patients' health and quality of life, the association of this outcome with the ACB has not been addressed by any prior systematic review. ${ }^{1-4}$ Moreover, prior systematic reviews mainly focused on the evaluation of methods for measuring the ACB.$^{59}{ }^{58}$ Conversely, other important aspects of study design have been hardly addressed to date.

To design this protocol for a systematic review on the $\mathrm{ACB}$ and the risk of fractures, we conducted a pilot evaluation of studies addressing this association. In this pilot evaluation, we identified five studies published between 2014 and 2017..$^{39-415960}$ Their results varied widely, with two studies showing no association, ${ }^{59} 60$ one reporting a risk increased by $14 \%^{39}$ and the other two studies reporting a risk increased by $56 \%{ }^{41}$ and by $71 \%,{ }^{40}$ respectively. Given the wide variability in risk observed in this pilot evaluation, there is a need to conduct a systematic review of studies on fractures and the anticholinergic burden. This systematic review aims to provide a better understanding of study characteristics that may explain the variability in risk estimates. These characteristics may include, for instance, the type and size of the population studied, its morbidity profile, its use of other medications that increase the risk of fractures. This systematic review aims also to identify specific subgroups at a particularly increased risk. Moreover, a summary measure of risk may be obtained, either for the overall population or for specific and more homogeneous subgroups, if the number of studies retrieved and their heterogeneity will allow the conduct of a meta-analysis.

The observed variability in risk estimates may be partly due to methodological differences among the studies conducted so far. From our pilot evaluation, it emerged that methodological differences may relate to the way exposure has been measured, for example, using different scales, ${ }^{39-41}$ or as the use of any MAC instead of as cumulative exposure. ${ }^{59}$ Other differences consist in outcome measurement, adjustment for potential confounders and other relevant factors (eg, only one study adjusted for several comorbidities specifically associated with the risk of fractures or with the use of MACs, ${ }^{39}$ while other studies adjusted only for aggregated indicators of morbidity, such as the Charlson Comorbidity Index ${ }^{40}$ or the Elixhauser score). ${ }^{41}$ Thus, potential confounders may not have been accounted for, leading to residual confounding. Moreover, some studies were conducted on individuals at greater risk of falls and fractures, such as patients with Parkinson's disease ${ }^{41}$ or nursing home residents. ${ }^{39}$ Therefore, it is unclear if their results are generalisable. Finally, one study ${ }^{60}$ included a small number of subjects and the precision of its point estimates may be low. Our pilot evaluation, although restricted to a pool of articles not identified systematically, shows the need of a comprehensive methodological evaluation of studies on the risk of fractures associated with the ACB. Such a methodological evaluation will provide guidance for future research.

\section{METHODS AND ANALYSES}

To fill the gaps in the knowledge presented above, we aim to conduct a systematic review on the methodological aspects of studies on the association between the ACB and the risk of fractures. We also aim to obtain a summary measure of the risk of fractures associated with the ACB, if the number of studies retrieved and their heterogeneity will allow us to perform a meta-analysis. This summary measure of risk may refer to the overall population or to specific and more homogeneous subgroups (eg, older adults). Additionally, we will evaluate the association between ACB and osteoporosis and/or reduced bone mineral density in studies that addressed fractures as an outcome.

The review will be reported based on Preferred Reporting Items of Systematic Reviews and Meta-Analyses Protocol guidelines. The protocol has been registered in the PROSPERO database and follows the Preferred Reporting Items of Systematic Reviews and Meta-Analyses Protocol, ${ }^{61}$ Meta-analysis of observational studies in epidemiology ${ }^{62}$ guidelines as well as a guideline for the conduct of systematic reviews and meta-analyses in older adults. ${ }^{63}$ We are planning to start the review in Q3 2019 and to finish it at the latest in Q2 2020.

\section{Sources of evidence}

Search strategies were developed by the project team under the guidance of an experienced medical librarian. 
Potentially eligible studies will be searched in the following electronic databases and information resources: MEDLINE (1950 to present), EMBASE (1947 to present) and Science Citation Index (1900 to present). Moreover, we will search in Cochrane Controlled Register of Trials (CENTRAL), sources dedicated to grey literature (Open Grey, OSFPreprints, GreyLit and Google Scholar) as well as hand searches in relevant open access repositories (Open DOAR).

\section{Search strategy}

The search strategy includes two concepts: anticholinergic (including medication and burden) and fractures (online supplementary appendix A). The appropriate controlled terms representing these concepts in each database will be used. To identify additional potentially eligible studies, we will manually screen the references of included studies and of prior systematic reviews or meta-analyses. We will also assess the relevance of articles that cite included articles. Additionally, we will contact authors who have published in this field for articles that may have been missed or are unpublished. To address the association between ACB and osteoporosis and/or reduced bone mineral density, we will conduct a second search. The search strategy will include the concepts anticholinergic (including medication and burden) and osteoporosis and/or reduced bone mineral density (online supplementary appendix A).

\section{Eligibility criteria}

To be included in this systematic review, studies have to fulfil each of the criteria outlined below.

\section{Study design}

Observational studies, such as cohort, case-control, case-crossover and self-controlled cohort studies.

\section{Population}

Studies conducted in human populations, without restrictions regarding the setting (ie, both population-based studies and studies including persons hospitalised or residents of nursing homes or other types of long-term care facility).

Since they are a high-risk group for fractures, some studies may include only older adults. However, we will not set an age limit as inclusion criteria nor restrict our systematic review to studies including only older adults. The reason is that we aim to evaluate the relation between $\mathrm{ACB}$ and fractures also in middle-aged and younger persons. It should be noted that some recent evidence suggests that the risk of fractures associated with ACB is increased in middle-aged women and not only in those 65 years or older. ${ }^{47}$

\section{Exposure}

Studies in which the ACB, as an exposure, was measured using a scale either previously published or newly developed. Studies that assessed cumulative exposure to MACs not based on a previously published list or scale will be included in the systematic review but not in the meta-analysis. On the contrary, studies that assessed the exposure to one or more individual MACs but did not assess any cumulative exposure to MACs or the ACB will be excluded from the systematic review.

\section{Outcome}

First, studies addressing fractures as an outcome without restriction to a defined site (ie, fractures of any site, eg, of the hip, of the hip and the femur, of the wrist) or to a defined type (ie, any fractures for whichever reason, eg, fall-related, fragility-related). Second, studies addressing osteoporosis and/or reduced bone mineral density.

\section{Measures of association}

Studies that report a crude or adjusted measure of the association between the exposure and the outcome (ie, relative risk, OR, HR or rate ratio), and the corresponding $95 \% \mathrm{CI}$, or that report sufficient data to calculate a measure of association.

\section{Data sources}

Studies based on any type of data, specifically, those conducted using primary collected data as well as those based on secondary data sources (eg, claims and other administrative health databases).

\section{Language}

No language restriction will be applied.

Time

No date restrictions will be applied.

\section{STUDY RECORDS}

\section{Data management}

Results of the literature search will be imported into an EndNote ${ }^{\odot}$ database and duplicates will be removed. Remaining articles will be entered into a Microsoft Access $2010^{\odot}$ database using structured data entry forms created specifically for this systematic review. Study information, including abstracts and full-text articles will be uploaded into the database (online supplementary appendix B). Before start of data collection, data entry forms will be piloted and the study team will receive training.

\section{Selection process}

Articles will be selected in a two-stage process. In the first step, the title and abstract of all articles identified by the search strategies will be assessed based on the eligibility criteria. Each article will be graded as eligible/not eligible/possibly eligible. In the second step, full texts of all eligible and possibly eligible articles will be retrieved and examined based on eligibility criteria to confirm their inclusion. Each article will be graded as either included or not included into the systematic review and the meta-analysis.

Both steps of the assessment will be performed independently by two reviewers (OR and JR). For each article, 
the evaluation of the two reviewers will be compared and discrepancies will be solved by consensus. In case a consensus cannot be reached, FEP (Co-PI) will mediate. Reasons for exclusion at both stages of the inclusion process will be recorded.

\section{Data extraction process}

Two reviewers (OR and JR) will extract data independently from full-text articles included for systematic review using the standardised MS Access data entry form mentioned above. Quality controls of the input data will be performed, including identification of missing data and discrepancies as well as logical checks of extracted data. Missing data, errors and discrepancies will be solved by re-evaluating the full-text article and/or by discussion between the reviewers and subsequent editing. If relevant information is missing from the full-text article, the corresponding author will be contacted and asked to provide clarification (up to three contact attempts by email).

\section{Risk of bias in individual studies}

The risk of bias for each included study will be assessed using two quality assessment tools: the Newcastle-Ottawa Quality Assessment Scale (NOS) ${ }^{64}$ and the RTI item bank. ${ }^{65}$ We chose to use two different tools to have a more in-depth understanding of the risk of bias in the included articles. The NOS is a widely used tool and covers the main aspects of study design. We added the RTI item bank because it evaluates certain aspects (eg, exposure assessment) with greater detail and it has been shown to be suited to assess observational drug safety studies in an ad-hoc tailored version that addressed bias particularly relevant for these types of studies (eg, indication bias, unmeasured confounding) ${ }^{66}$ Given that this systematic review will include observational studies on the safety of medications as well, we considered the RTI item bank an appropriate additional tool.

The NOS was developed for the assessment of the risk of bias in case-control and cohort studies. It assesses the risk of bias by means of a brief questionnaire that evaluates specific aspects of the study design. For case-control studies, the questionnaire covers the following domains: methods for selection of cases and controls, the comparability of cases and controls as well as the methods used for ascertainment of the exposure. The domains for cohort studies are methods for selection of the cohort, comparability of exposed and non-exposed participants and the methods for the assessment of outcomes. Studies are then rated according to their estimated risk of bias.

The RTI item bank was developed for evaluating the quality of observational studies with focus on bias and precision. It consists of 29 questions and covers 11 domains: sample definition and selection, interventions/exposure, outcomes, creation of treatment groups, blinding, soundness of information, follow-up, analysis comparability, analysis outcome, interpretation and presentation and reporting. In each question, the risk of bias is rated as low, unclear or high risk of bias.
Results will be presented for each item for each study and as an overall risk of bias for each study. The questions of the RTI item bank will be tailored to evaluate aspects of study design and analysis that are specifically relevant to the research question of this systematic review.

Each included study will be independently assessed by each reviewer (OR and JR) using the NOS and RTI item bank. For each item of each tool, disagreement between the ratings of reviewers will be solved by consensus. If consensus cannot be reached, FEP will be consulted. In the case of insufficient information reported in the study, the risk of bias will be judged 'unclear' and the corresponding author of the study will be contacted and asked to provide clarification (up to three contact attempts by email).

\section{Data synthesis}

Relevant characteristics of excluded and included studies will be summarised through statistical measures as appropriate (eg, percentages, mean, median, SD, IQR) and tabulated.

Between-study heterogeneity will be assessed through appropriate statistics including the $Q$ statistic. ${ }^{67}$ The proportion of total between-studies variance due to heterogeneity will be estimated, for example, through the $\mathrm{I}^{2}$ statistic. ${ }^{68}$ Studies that influence the heterogeneity will be detected ${ }^{69}$ and sources of heterogeneity will be explored. Sensitivity analysis will be conducted removing studies that influence heterogeneity. In the presence of relevant heterogeneity, the summary relative risk and its 95\% CI will be calculated using a random-effects model. The effect of potential covariates on the summary relative risk will be assessed. We will use meta-regression to assess the influence of the scale used in the different studies to evaluate the ACB. If a quantitative synthesis is not possible, a qualitative assessment of the results will be conducted. We will perform a leave-one-out sensitivity analysis, iteratively removing one study at a time, to measure how each single study affects the overall estimate and to identify studies that potentially drive the results. ${ }^{70}$ Other sensitivity analyses will include the omission of specific studies (eg, those at high risk of bias), the restriction to specific subgroups (eg, more homogeneous studies) or studies that share definite characteristics (eg, include only women or only older adults) and the stratification by age. Finally, we will report the number and percentage of studies that considered the outcome bone density and summarise their results, either in a quantitative or a narrative way (if a low number of studies are found).

Publication bias will be assessed graphically by means of a funnel-plot and using Egger's test. ${ }^{71} 72$ If publication bias is detected, appropriate methods such as the trimand-fill method will be used to correct the bias. ${ }^{73}$

Acknowledgements The publication of this article was funded by the 0pen Access Fund of the Leibniz Association. 
Contributors JR: study design, conduct of study, bibliographic research, design of data entry forms, data management, protocol and manuscript writing and review. WS: protocol and manuscript review. LC: bibliographic research design and conduct, protocol and manuscript review. FB-A: study design, protocol and manuscript review. OR: study conception and design, scientific coordination, protocol and manuscript review. FEP: study conception and design, scientific coordination, protocol and manuscript writing and review.

Funding This systematic review was funded entirely by internal funds of the Leibniz Institute for Prevention Research and Epidemiology: BIPS. The funding institution had no influence on any part of this protocol.

Competing interests None declared.

Patient and public involvement statement This systematic review protocol was done without patient involvement. Patients were not invited to comment on the study design or invited to contribute to the writing or editing of this document for readability or accuracy.

\section{Patient consent for publication Not required.}

Ethics approval No ethics approval will be sought, as no original data will be collected for this review. Findings will be disseminated through peer-reviewed publication and conference presentations.

Provenance and peer review Not commissioned; externally peer reviewed.

Open access This is an open access article distributed in accordance with the Creative Commons Attribution Non Commercial (CC BY-NC 4.0) license, which permits others to distribute, remix, adapt, build upon this work non-commercially, and license their derivative works on different terms, provided the original work is properly cited, appropriate credit is given, any changes made indicated, and the use is non-commercial. See: http://creativecommons.org/licenses/by-nc/4.0/.

\section{REFERENCES}

1. Cardwell K, Hughes CM, Ryan C. The Association Between Anticholinergic Medication Burden and Health Related Outcomes in the 'Oldest Old': A Systematic Review of the Literature. Drugs Aging 2015;32:835-48

2. Fox C, Smith T, Maidment I, et al. Effect of medications with anti-cholinergic properties on cognitive function, delirium, physical function and mortality: a systematic review. Age Ageing 2014;43:604-15.

3. Salahudeen MS, Chyou T-yuan, Nishtala PS. Serum anticholinergic activity and cognitive and functional adverse outcomes in older people: a systematic review and meta-analysis of the literature. PLoS One 2016;11:e0151084.

4. Salahudeen MS, Duffull SB, Nishtala PS. Anticholinergic burden quantified by anticholinergic risk scales and adverse outcomes in older people: a systematic review. BMC Geriatr 2015;15:31.

5. Durán CE, Azermai M, Vander Stichele RH. Systematic review of anticholinergic risk scales in older adults. Eur J Clin Pharmacol 2013;69:1485-96.

6. Lieberman JA. Managing anticholinergic side effects. Prim Care Companion J Clin Psychiatry 2004;6(Suppl 2):20-3.

7. de Leon J. Paying attention to pharmacokinetic and pharmacodynamic mechanisms to progress in the area of anticholinergic use in geriatric patients. Curr Drug Metab 2011;12:635-46.

8. Tune LE. Anticholinergic effects of medication in elderly patients. $J$ Clin Psychiatry 2001;62 Suppl 21(Suppl 21):11-14.

9. Mayer T, Haefeli WE, Seidling HM. Different methods, different results - how do available methods link a patient's anticholinergic load with adverse outcomes? Eur J Clin Pharmacol 2015;71:1299-314

10. Briet J, Javelot H, Heitzmann $\mathrm{E}$, et al. The anticholinergic impregnation scale: towards the elaboration of a scale adapted to prescriptions in French psychiatric settings. Therapies 2017;72:427-37.

11. Klamer TT, Wauters M, Azermai M, et al. A novel scale linking potency and dosage to estimate anticholinergic exposure in older adults: the muscarinic acetylcholinergic receptor antagonist exposure scale. Basic Clin Pharmacol Toxicol 2017;120:582-90.

12. Gray SL, Anderson ML, Dublin S, et al. Cumulative use of strong anticholinergics and incident dementia: a prospective cohort study. JAMA Intern Med 2015;175:401-7.

13. Hilmer SNet al. A drug burden index to define the functional burden of medications in older people. Arch Intern Med 2007;167:781-7.
14. Ancelin ML, Artero S, Portet F, et al. Non-degenerative mild cognitive impairment in elderly people and use of anticholinergic drugs: longitudinal cohort study. BMJ 2006;332:455-9.

15. Campbell NL, Boustani MA, Lane KA, et al. Use of anticholinergics and the risk of cognitive impairment in an African American population. Neurology 2010;75:152-9.

16. Campbell NL, Perkins AJ, Bradt P, et al. Association of anticholinergic burden with cognitive impairment and health care utilization among a diverse ambulatory older adult population. Pharmacotherapy 2016;36:1123-31.

17. Carrière I, Fourrier-Reglat A, Dartigues J-F, et al. Drugs with anticholinergic properties, cognitive decline, and dementia in an elderly general population. Arch Intern Med 2009;169:1317-24.

18. Ehrt U, Broich K, Larsen JP, et al. Use of drugs with anticholinergic effect and impact on cognition in Parkinson's disease: a cohort study. Journal of Neurology, Neurosurgery \& Psychiatry 2010;81:160-5

19. Fox C, Richardson K, Maidment ID, et al. Anticholinergic medication use and cognitive impairment in the older population: the medical Research Council cognitive function and ageing study. J Am Geriatr Soc 2011;59:1477-83.

20. Han L, Agostini JV, Allore HG. Cumulative anticholinergic exposure is associated with poor memory and executive function in older men. $J$ Am Geriatr Soc 2008;56:2203-10.

21. Rudolph JLet al. The anticholinergic risk scale and anticholinergic adverse effects in older persons. Arch Intern Med 2008;168:508-13.

22. Lowry E, Woodman RJ, Soiza RL, et al. Associations between the anticholinergic risk scale score and physical function: potentia implications for adverse outcomes in older hospitalized patients. $J$ Am Med Dir Assoc 2011;12:565-72.

23. Lowry E, Woodman RJ, Soiza RL, et al. Drug burden index, physical function, and adverse outcomes in older hospitalized patients. J Clin Pharmacol 2012;52:1584-91.

24. Boccardi V, Baroni M, Paolacci L, et al. Anticholinergic burden and functional status in older people with cognitive impairment: results from the ReGAI project. J Nutr Health Aging 2017;21:389-96.

25. Lampela P, Lavikainen P, Garcia-Horsman JA, et al. Anticholinergic drug use, serum anticholinergic activity, and adverse drug events among older people: a population-based study. Drugs Aging 2013;30:321-30.

26. Richardson K, Bennett K, Maidment ID, et al. Use of medications with anticholinergic activity and self-reported injurious falls in older community-dwelling adults. J Am Geriatr Soc 2015;63:1561-9.

27. Zimmerman KM, Salow M, Skarf LM, et al. Increasing anticholinergic burden and delirium in palliative care inpatients. Palliat Med 2014;28:335-41.

28. Han L, McCusker J, Cole M, et al. Use of medications with anticholinergic effect predicts clinical severity of delirium symptoms in older medical inpatients. Arch Intern Med 2001;161:1099-105.

29. Myint PK, Fox C, Kwok CS, et al. Total anticholinergic burden and risk of mortality and cardiovascular disease over 10 years in 21,636 middle-aged and older men and women of EPIC-Norfolk prospective population study. Age Ageing 2015;44:219-25.

30. Collamati A, Martone AM, Poscia A, et al. Anticholinergic drugs and negative outcomes in the older population: from biological plausibility to clinical evidence. Aging Clin Exp Res 2016;28:25-35.

31. Mintzer J, Burns A. Anticholinergic side-effects of drugs in elderly people. J R Soc Med 2000;93:457-62.

32. Chatterjee $\mathrm{S}$, Carnahan RM, Chen $\mathrm{H}$, et al. Anticholinergic medication use and risk of pneumonia in elderly adults: a nested case-control study. J Am Geriatr Soc 2016;64:394-400.

33. Lampela P, Tolppanen A-M, Tanskanen A, et al. Anticholinergic Exposure and Risk of Pneumonia in Persons with Alzheimer's Disease: A Nested Case-Control Study. Journal of Alzheimer's Disease 2017;56:119-28.

34. Pisa FE, Verriello L, Deroma L, et al. The accuracy of discharge diagnosis coding for amyotrophic lateral sclerosis in a large teaching hospital. Eur J Epidemiol 2009;24:635-40.

35. Villanueva CM, Gracia-Lavedan E, Bosetti C, et al. Colorectal cancer and long-term exposure to Trihalomethanes in drinking water: a multicenter Case-Control study in Spain and Italy. Environ Health Perspect 2017;125:56-65.

36. Marcum ZA, Perera S, Thorpe JM, et al. Anticholinergic use and recurrent falls in community-dwelling older adults: findings from the health ABC study. The Annals of pharmacotherapy 2015;49:1214-21.

37. Marcum ZA, Wirtz HS, Pettinger M, et al. Anticholinergic medication use and falls in postmenopausal women: findings from the women's health initiative cohort study. BMC Geriatr 2016;16:76.

38. Wauters M, Klamer T, Elseviers M, et al. Anticholinergic exposure in a cohort of adults aged 80 years and over: associations of the 
MARANTE scale with mortality and hospitalization. Basic Clin Pharmacol Toxicol 2017;120:591-600.

39. Chatterjee S, Bali V, Carnahan RM, et al. Anticholinergic medication use and risk of fracture in elderly adults with depression. $J$ Am Geriatr Soc 2016;64:1492-7.

40. Hsu W-H, Wen Y-W, Chen L-K, et al. Comparative associations between measures of Anti-cholinergic burden and adverse clinical outcomes. Ann Fam Med 2017;15:561-9.

41. Crispo JAG, Willis AW, Thibault DP, et al. Associations between anticholinergic burden and adverse health outcomes in Parkinson disease. PLOS One 2016;11:e0150621.

42. Ambrose AF, Cruz L, Paul G. Falls and fractures: a systematic approach to screening and prevention. Maturitas 2015;82:85-93.

43. Seppala LJ, van der Velde N, Masud T, et al. EuGMS task and Finish group on Fall-Risk-Increasing drugs (FRIDs): position on knowledge Dissemination, management, and future research. Drugs Aging 2019;36:299-307.

44. de Vries M, Seppala LJ, Daams JG, et al. Fall-Risk-Increasing drugs: a systematic review and meta-analysis: I. cardiovascular drugs. J Am Med Dir Assoc 2018;19:371.e1-371.e9.

45. Seppala LJ, van de Glind EMM, Daams JG, et al. Fall-Risk-Increasing drugs: a systematic review and meta-analysis: III. others. J Am Med Dir Assoc 2018;19:372.e1-372.e8.

46. Seppala LJ, Wermelink AMAT, de Vries M, et al. Fall-Risk-Increasing drugs: a systematic review and meta-analysis: II. psychotropics. $J$ Am Med Dir Assoc 2018;19:371.e11-371.e17.

47. Ablett AD, Wood AD, Barr R, et al. A high anticholinergic burden is associated with a history of falls in the previous year in middleaged women: findings from the Aberdeen prospective osteoporosis screening study. Ann Epidemiol 2018;28:557-62.

48. Tajeu GS, Delzell E, Smith W, et al. Death, debility, and destitution following hip fracture. J Gerontol A Biol Sci Med Sci 2014;69A:346-53.

49. Tang VL, Sudore R, Cenzer IS, et al. Rates of recovery to pre-fracture function in older persons with hip fracture: an observational study. $J$ Gen Intern Med 2017;32:153-8.

50. Bliuc Det al. Mortality risk associated with low-trauma osteoporotic fracture and subsequent fracture in men and women. JAMA 2009;301:513-21.

51. Klop C, van Staa TP, Cooper C, et al. The epidemiology of mortality after fracture in England: variation by age, sex, time, geographic location, and ethnicity. Osteoporos Int 2017;28:161-8.

52. Katsoulis M, Benetou V, Karapetyan T, et al. Excess mortality after hip fracture in elderly persons from Europe and the USA: the chances project. J Intern Med 2017;281:300-10.

53. Bleibler F, Rapp K, Jaensch A, et al. Expected lifetime numbers and costs of fractures in postmenopausal women with and without osteoporosis in Germany: a discrete event simulation model. BMC Health Serv Res 2014;14:284.

54. Lambrelli D, Burge R, Raluy-Callado M, et al. Retrospective database study to assess the economic impact of hip fracture in the United Kingdom. J Med Econ 2014;17:817-25.

55. Leal J, Gray AM, Prieto-Alhambra D, et al. Impact of hip fracture on hospital care costs: a population-based study. Osteoporos Int 2016;27:549-58.
56. Ruxton K, Woodman RJ, Mangoni AA. Drugs with anticholinergic effects and cognitive impairment, falls and all-cause mortality in older adults: a systematic review and meta-analysis. $\mathrm{Br} J$ Clin Pharmacol 2015;80:209-20.

57. Welsh TJ, van der Wardt V, Ojo G, et al. Anticholinergic drug burden Tools/Scales and adverse outcomes in different clinical settings: a systematic review of reviews. Drugs Aging 2018;35:523-38.

58. Wouters $\mathrm{H}$, van der Meer H, Taxis K. Quantification of anticholinergic and sedative drug load with the drug burden index: a review of outcomes and methodological quality of studies. Eur $\mathrm{J}$ Clin Pharmacol 2017;73:257-66.

59. Marcum ZA, Wirtz HS, Pettinger M, et al. Anticholinergic Medication Use and Fractures in Postmenopausal Women: Findings from the Women's Health Initiative. Drugs Aging 2015;32:755-63.

60. Fraser L-A, Adachi JD, Leslie WD, et al. Effect of anticholinergic medications on falls, fracture risk, and bone mineral density over a 10-year period. Ann Pharmacother 2014;48:954-61.

61. Moher D, Shamseer L, Clarke M, et al. Preferred reporting items for systematic review and meta-analysis protocols (PRISMA-P) 2015 statement. Syst Rev 2015;4:1.

62. Stroup DF, Berlin JA, Morton SC, et al. Meta-Analysis of observational studies in epidemiology: a proposal for reporting. meta-analysis of observational studies in epidemiology (moose) group. JAMA 2000;283:2008-12.

63. Shenkin SD, Harrison JK, Wilkinson T, et al. Systematic reviews: guidance relevant for studies of older people. Age Ageing 2017;46:722-8.

64. Wells G, Shea B, Oconnell D. The Newcastle-Ottawa scale (NOS) for assessing the quality of nonrandomized studies in meta-analyses. Available: http://www.ohri.ca/programs/clinical_epidemiology/oxford. asp [Accessed 22 Aug 2018].

65. Viswanathan M, Berkman ND. Development of the RTI item bank on risk of bias and precision of observational studies. J Clin Epidemiol 2012;65:163-78.

66. Margulis AV, Pladevall M, Riera-Guardia N, et al. Quality assessment of observational studies in a drug-safety systematic review, comparison of two tools: the Newcastle-Ottawa scale and the RTI item bank. Clin Epidemiol 2014;6:359-68.

67. Whitehead A, Whitehead J. A general parametric approach to the meta-analysis of randomized clinical trials. Stat Med 1991;10:1665-77.

68. Higgins JPT, Thompson SG. Quantifying heterogeneity in a metaanalysis. Stat Med 2002;21:1539-58.

69. Viechtbauer W, Cheung MW-L. Outlier and influence diagnostics for meta-analysis. Res. Synth. Method 2010;1:112-25.

70. Salvi V, Grua I, Cerveri G, et al. The risk of new-onset diabetes in antidepressant users - A systematic review and meta-analysis. PLOS One 2017;12:e0182088.

71. Egger M, Smith GD, Schneider M, et al. Bias in meta-analysis detected by a simple, graphical test. BMJ 1997;315:629-34.

72. Egger M, Schneider M, Smith GD. Meta-Analysis spurious precision? meta-analysis of observational studies. BMJ 1998;316:140-4.

73. Duval S, Tweedie R. Trim and fill: a simple funnel-plot-based method of testing and adjusting for publication bias in meta-analysis. Biometrics 2000;56:455-63. 